\title{
Peter Schmitz
}

\section{»Der einzige Einwanderer nach Schlesien«: Gregor Lange und das Breslauer Musikleben des späten I6. Jahrhunderts}

Der Protagonist dieses Beitrages, der um I545 im brandenburgischen Havelberg geborene Gregor Lange, galt im späten I6. Jahrhundert als ein Tonkünstler von durchaus hohem Rang. In einem 595 erschienenen Liederbuch von Christoph Demantius wird er etwa mit Orlando di Lasso in Verbindung gebracht und gewissermaßen mit Leonhard Lechner auf eine Stufe gestellt: »[...] Orlandus valuit permultum cantibus olim, Langius et Lechner non valuere minus [...] « (Epigramm des Heilbronners Andreas Wecker). ${ }^{1}$ So ehrenvoll eine solche Würdigung auch anmuten mag, sie scheint recht bald verhallt zu sein. Neben dem fünfstimmigen geistlichen Gesang Ich will des Herren Zorn tragen (nach Micha 7,9), der z.B. in mehreren Handschriften der Sammlung Löbau in der Sächsischen Landesbibliothek Dresden ${ }^{2}$ überliefert ist, scheinen ab der Mitte des I7. Jahrhunderts allenfalls noch Langes dreistimmige weltliche Lieder - etwa in Umarbeitungen von Demantius und Ambrosius Profe - zum gepflegten Repertoire gezählt zu haben. Robert Eitner wies denn auch I883 darauf hin, dass »die neuere Zeit noch wenig Notiz« von dem Komponisten genommen habe. ${ }^{3}$ Dass sich im Nachlass des in München wirkenden Komponisten Josef Gabriel Rheinberger die Abschrift einer sechsstimmigen Motette Langes findet, kann für das I9. Jahrhundert eher als

I Zitiert nach Christoph Demantius, Neue Teutsche Weltliche Lieder I595, hrsg. von Kurt Stangl (Das Erbe deutscher Musik, Sonderreihe, I), Kassel I954, S. 4.

2 Mus.Löb.8 + 70; Mus.Löb.IO; Mus.Löb.3I; Mus.Löb.42; Mus.Löb.5I; Mus.Löb.53; Mus.Löb.54 Siehe auch: Mus.I-D-503 und Mus.Sche35. Vgl. dazu Wolfgang Steude, Die Musiksammelhandschriften des 16. und 17. Jahrhunderts in der Sächsischen Landesbibliothek zu Dresden (Quellenkataloge zur Musikgeschichte, 6), Leipzig I974, S.36, 60, II2, I22, I34, I39, I46, I48, I55, 215 Zur Überlieferung dieses Werkes in der Breslauer Stadtbibliothek und der Liegnitzer Bibliotheca Rudolfina vgl. Reinhold Starke, »Hieronymus Gregorius Langius Havelbergensis «, in: Monatshefte für Musikgeschichte 3I (I899), S. IOI-IIO, II3-I23, hier S. I22.

3 Dies stünde im Widerspruch zur reichen handschriftlichen Überlieferung seiner Werke. Vgl. Robert Eitner, Art. »Gregor Lange«, in: Allgemeine Deutsche Biographie, auf Veranlassung Seiner Majestät des Königs von Bayern hrsg. durch die historische Commission bei der Königlichen Akademie der Wissenschaften, Bd. I7 (I883), Reprint Berlin I969, S. 632. 
Ausnahme gelten. ${ }^{4}$ In der Folge fehlte es freilich nicht an Anstrengungen, Lange wieder ins Gedächtnis zu rufen. Allen voran Reinhold Starke trug durch eine in den Monatsheften für Musikgeschichte im Jahr I899 ${ }^{5}$ zum Abdruck gelangten Studie zur Erhellung der Biographie Langes bei, indem er manche Ergänzungen und Korrekturen zu den spärlichen lexikographischen Angaben bei Walther (I732), Gerber (I790 bzw. I8I3), ${ }^{7}$ Schilling (I837) ${ }^{8}$ und Fétis (I870) ${ }^{9}$ lieferte. Auch gab er im Jahr I90I eine Auswahl vier- bis achtstimmiger Motetten des Komponisten heraus. ${ }^{10}$ Das erklärte Ziel Starkes war es, das »Anrecht «, welches Lange nach seiner Überzeugung habe, »als der Dritte im Bunde mit Orlandus Lassus und Leonhard Lechner genannt zu werden, zu beweisen. «11

Anhand des Werdeganges einer Einzelgestalt übergreifende epochale Prozesse aufzuzeigen, ist nicht nur in der Musikwissenschaft ein beliebter und verbreiteter Ansatz. Dass die Wahl für eine solch exemplarische Darstellung gerade auf Gregor Lange fiel, mag angesichts des titelgebenden Zitats von Lothar Hoffmann-Erbrecht überraschen. Lange sei der »einzige Einwanderer nach Schlesien«. ${ }^{2}$ Damit wird ja gerade das Singuläre seiner Person betont und suggeriert, dass seine Biographie im Grunde keine Vergleichsebenen anbiete. Zur Einordnung: Das Zitat spielt auf den Umstand an, dass der Komponist, nachdem er als Kantor an der schola senatoria in Frankfurt/Oder (seit 1574) gewirkt hatte, I583 nach Breslau übersiedelte. Auch wenn Lange hier offenbar keine feste Stellung inne hatte und - wie gleich noch zu zeigen sein wird - sein dortiges Wirken v. a. von Krankheit überschattet war, ${ }^{13}$ gestalteten sich die im kulturellen Zentrums Schlesiens verbrachten letzten Lebensjahre in künstlerischer Sicht doch ausgesprochen produktiv. Diese Konstellation erweist sich für das Generalthema des Symposiums, das ja insbesondere musikalische Transferprozesse in den Blick nimmt, als durchaus ergiebig. Ausgehend von der Person Langes wäre folglich nach Verschränkungen der Kulturräume Kurbrandenburgs und Schlesiens zu fragen.

4 Ego dormio et cor meum vigilat, Datierung der Abschrift: »den 5/I2 8I«. Bayerische Staatsbibliothek, Musikabteilung, München, D-Mbs Mus. ms. 4746-33.

5 Vgl. die bibliographische Angabe in Anm. 2.

6 Johann Gottfried Walther, Musicalisches Lexicon oder Musicalische Bibliothec, Leipzig I732, S. 354.

7 Ernst Ludwig Gerber, Historisch-biographisches Lexicon der Tonkünstler, Bd. I, Leipzig I790, Sp. 785 sowie ders., Nemes historisch-biographisches Lexicon der Tonkünstler, Bd. 3, Leipzig I8I3, Sp. I7O.

8 Gustav Schilling, Encyclopädie der gesammten musikalischen Wissenschaften, oder Universal-Lexicon der Tonkunst, Bd. 4, Stuttgart I837, S. 3 I7.

9 François-Joseph Fétis, Biographie universelle des musiciens et bibliographie générale de la musique, Bd. 5, Paris ${ }^{2}$ I870, S. I90.

IO Gregor Langius, Eine ausgewäblte Sammlung Motetten zu 4, s, 6 und 8 Stimmen. In Partitur gesetzt und mit einer Klavierpartitur versehen von Reinhold Starke (Publikation älterer praktischer und theoretischer Musikwerke, 25), Leipzig I9OI.

I I Starke, »Hieronymus Gregorius Langius Havelbergensis« (wie Anm. 2), S. IO2.

I2 Lothar Hoffmann-Erbrecht, Musikgeschichte Schlesiens (Die Musik der Deutschen im Osten Mitteleuropas, I), Dülmen I986, S. 63.

I3 Er verstarb bereits vier Jahre später 1587. 
Ob Lange tatsächlich der einzige brandenburgische Komponist war, der im späten I6. Jahrhundert nach Schlesien auswanderte, ist kaum zu beurteilen. In jedem Fall dürfte es sich dabei aber um eine Ausnahme gehandelt haben. ${ }^{14}$ Dass er gerade nach Breslau übersiedelte, überrascht indes nicht: Die kulturellen und wirtschaftlichen Beziehungen zwischen beiden Städten waren im I6. Jahrhundert überaus intensiv. Gottfried Kliesch hat anhand von Matrikeln insgesamt $599 \mathrm{Na}$ men von Breslauern bzw. Wahlbreslauern ermitteln können, die an der Alma Mater Viadrina zu Frankfurt im Zeitraum zwischen 1506 und I648 studiert haben. ${ }^{15}$ Darunter sind auch einige Musikerpersönlichkeiten wie der spätere Breslauer Kantor Simon Besler nachzuweisen. ${ }^{16}$ Und auch im Lehrköper der Oderuniversität, die im »Dreierverbund von Landesfürst, Ständen, v. a. der Landeskirche, und der Universitätsstadt bzw. Angehörigen ihrer städtischen Führungsschicht ${ }^{17}$ finanziell getragen wurde, finden sich in der zweiten Jahrhunderthälfte einige aus Schlesien stammende Persönlichkeiten, so etwa der protestantische Theologe Christoph Pelargus. Lange, der sich zum Sommersemester I573 an der Viadrina immatrikulierte und wie erwähnt bereits im Folgejahr durch den Rat der Stadt zum Kantor ernannt wurde, verfügte nachweislich bereits in seiner Frankfurter Zeit über Verbindungen zu Breslauern. ${ }^{18}$ Ein solcher Kontakt wird in der Vorrede zu den 1580 publizierten Cantiones aliquot novae ${ }^{19}$ mit dem Kaufmann Antonius Zehentener

I4 Auch der aus Franken stammende Thomas Elsbeth hatte in Frankfurt/Oder studiert und ging im Anschluss nach Breslau.

I5 Vgl. Gottfried Kliesch, Der Einfluß der Universität Frankfurt (Oder) auf die schlesisches Bildungsgeschichte dargestellt an den Breslauer Immatrikulierten von I506-I648 (Quellen und Darstellungen zur schlesischen Geschichte, 5), Würzburg I96I, Teil 2, S. 75-87.

I6 Auch der Mathematiker und Musiker David Wolkenstein studierte an der Viadrina. Vgl. ebd., S. I95. Innerhalb der Artistischen (Philosophischen) Fakultät wurde Musik wie üblich als Teil des Quadriviums gelehrt.

I 7 Rainer Christoph Schwinges, »Die Universitätsgründung als Wirtschaftsfaktor im späten Mittelalter«, in: Universität und Stadt. Ringvorlesung zum 500. Jubiläum der Europa-Universität Viadrina Frankfurt (Oder), hrsg. von Ulrich Knefelkamp, Schöneiche bei Berlin 2007, S. 3I. I8II wurde die Viadrina nach Breslau verlegt.

I 8 U. a. hatte er zu dem aus Breslau stammenden David Rhenisch Kontakt. Zum studentischen Freundeskreis vgl. Heinrich Grimm, Meister der Renaissancemusik an der Viadrina. Quellenbeiträge zur Geisteskultur des Nordosten Deutschlands vor dem Dreißigjährigen Kriege, Frankfurt/Oder I942, S. I95. Ein Exemplar des Lange-Druckes Bekenntnis der Sünden, und Gebet umb gnedige Linderung der vorstehenden Not und Gefahr (Breslau 1585) trägt eine handschriftliche Dedikation des Autors an Rhenisch. Vgl. dazu Emil Bohn, Die musikalischen Handschriften des XVI. und XVII. Jahrhunderts in der Stadtbibliothek zu Breslau. Ein Beitrag zur Geschichte der Musik im XVI. und XVII. Jahrhundert, Breslau I890, S. 233.

I9 Gregor Lange, CANTIONES ALIQVOT NOVAE QVINQVE ET SEX VOCVM, TVM VIVA VOCE, TVM OMNIS GENERIS INSTRVMENTIS CANTATV COMMODISSIMAE Iam primum in lucem editae AVCTORE GREGORIO LANGIO HAVELBERGENSI. Cum gratia \& prinilegio Caesa: Maiest., Frankfurt/Oder I580. 
genannt. ${ }^{20}$ Das darin enthaltene, auf Psalm II9 basierende fünfstimmige Memor esto verbi tui ${ }^{21}$ (No. IO) ist dem Mäzen persönlich gewidmet. ${ }^{22}$ In dem bei Andreas Eichhorn ${ }^{23}$ in Frankfurt erschienenen Druck gibt es übrigens auch erste Hinweise auf Langes angeschlagenen Gesundheitszustand. ${ }^{24}$ Eine Kirchenrechnung von St. Marien aus dem gleichen Jahr (9. Juni I580) nennt ihn den »lamen Cantor «, 25 später wird es heißen, seine Hände und Füße seien »contract ${ }^{26}{ }^{6}$ Was zu der Erkrankung geführt hat, ist nicht bekannt. Jedenfalls dürfte sie ihn recht bald zur Aufgabe seiner Ämter in Frankfurt gezwungen haben. Die verbreitete Annahme, dass ihn Gönner wie der eben erwähnte Breslauer Kaufmann zur Übersiedlung veranlasst haben, erscheint keineswegs abwegig. Vermutlich waren damals bereits einige Arbeiten des Komponisten bekannt, im Druck war bis dato allerdings erst eine Hochzeitsmusik aus dem Jahr 1574 erschienen. ${ }^{27}$

Verweilen wir noch einen Moment in Frankfurt und beleuchten Langes dortige Sozialisation: Neben seinen Kontakten zu (heute weitgehend der Vergessenheit anheimgefallenen) Musikern wie den Organisten Christoph Zacharias und Georg Bruck sowie dem Komponisten Joachim Belitz, ${ }^{28}$ der im Übrigen ebenfalls in Breslauer Handschriften ${ }^{29}$ mit einigen Werken vertreten ist, verkehrte er auch im Umfeld namhafter Theologen. Allen voran zählte der kurbrandenburgische Gene-

20 "Quos tamen mea tenuitas nullo modo ferre potuisset, nisi vir honestus Antonius Zehentenerus Ciuis V. M. \& Musicae studio optime cupiens, mea inopiae auxilio fuißet.«

2 I Von den prominenten Kompositionen Josquin Desprez' und Giovanni Pierluigi da Palestrinas einmal abgesehen, handelt es sich um einen vergleichsweise selten vertonten Text.

22 Widmung in der Tenorstimme: »D. Anthonio Zehenenero dedicatum«.

23 Zur Bedeutung dieses Verlegers, der u. a. mehrere Lautentabulaturen drucklegen ließ, vgl. Ingeborg Allihn, Art. »Frankfurt an der Oder", in: Die Musik in Geschichte und Gegenwart, 2. neubearb. Ausgabe, hrsg. von Ludwig Finscher, Sachteil Bd. 3, Kassel 1995, Sp. 667. Bei Eichhorn erschien I584 die Tabulatura nova des schlesischen Lautenisten Gregor Krengel (ebenfalls ein Student der Viadrina). Darin finden sich u. a. mehrere Lautenintavolierungen von Werken Gregor Langes. Vgl. dazu Lothar Hoffmann-Erbrecht, »Schlesische Lautenisten in Mitteldeutschland «, in Kulturgeschichte Schlesiens in der Frühen Neuzeit Bd. 2, hrsg. von Klaus Garber, Tübingen 2005 S. 933-940, hier S. 934.

$24 »[\ldots]$ tantisper dum insperata calamiate non sine bonorum virorum commiseratione oppressus, ab officio me abdicarim.«

25 Zitiert nach Grimm, Meister der Renaissancemusik (wie Anm. I8), S. 192.

26 Zitiert nach Schilling, Encyclopädie der gesammten musikalischen Wissenschaften (wie Anm. 8), S. 317 .

27 Gregor Lange, Gamedion: in honorem pietate atgue virtute, ornatissimi viri, D. Adami Bolferosii, Frankfurt/Oder 1574.

28 Vgl. zum Frankfurter Bekanntenkreis, dem auch Johann Honeburg der Jüngere angehörte: Grimm, Meister der Renaissancemusik (wie Anm. I8), S. 195.

29 Z.B. in der Sammelhandschrift Ms.Mus. I5 aus den ehemaligen Beständen der Breslauer Stadtbibliothek (heute in der Musikabteilung der Staatsbibliothek zu Berlin). Vgl. dazu Bohn, Die musikalischen Handschriften (wie Anm. I8), S. 43-52. Zur Breslauer Stadtbibliothek siehe u. a. Ernst Wermke, »Zur Entstehung der Breslauer Stadtbibliothek «, in: Beitrüge zur Geschichte der Stadt Breslau 7 (1939), S. 39-47, und Alfred Rüffler, Die Stadtbibliothek Breslan im Spiegel der Erinnerung. Geschichte - Bestände - Forschungsstätte, Sigmaringen 1997. 
ralsuperintendent und Universitätsprofessor Andreas Musculus zu den Förderern Langes und spielte wohl auch bei der erwähnten Berufung in das Kantorenamt ein Rolle. ${ }^{30}$ Musculus' Name verbindet sich u.a. mit der sogenannten Teufelsliteratur: Hosentenfel (I555), Ehetenfel (I556), Fluchteufel (I556) und Des Teufels Tyrannei (I56I) sind einschlägige Publikationen von ihm innerhalb dieses Genres. Mit Blick auf die Musik sind nicht zuletzt seine I553 bzw. I559 erschienenen Gebetbücher relevant, da sie im I7. Jahrhundert offenbar für Vertonungen von Heinrich Schütz (Cantiones sacrae von 1625 und Kleine Geistliche Konzerte II von I639) und Dieterich Buxtehude (Quemadmodum desiderat cervus BuxWV 92) herangezogen wurden. ${ }^{31}$ Als dezidierter Akt der musikalischen Memoria versteht sich Langes vierstimmige Motette Media vita in morte sumus, die er zum Tode des Theologen I58I komponierte. Der Verstorbene, der seine eigenen Gedanken über Tod und Vergänglichkeit in Trostbüchern ${ }^{32}$ sowie der Schrift Bedencks Ende (Frankfurt/Oder I572) dargelegt hat, wird in der bereits erwähnten Breslauer Handschrift Ms. Mus. $15^{33}$ namentlich genannt: »in obitum d. A. Musculi«. Die lateinische einstrophige Antiphon Media vita in morte sumus aus dem II. Jahrhundert wurde bekanntlich I524 von Luther ins Deutsche übertragen, auf drei Strophen erweitert und musikalisch bearbeitet. ${ }^{34}$ In dieser Form - also mit dem deutschen Text »Mitten wir im Leben sind « - fand das Lied verschiedentlich Eingang in Gesangbücher (Erfurter Enchiridion I524, Johann Walters Chorgesangbuch I524 u. a.). Allerdings sind schon aus vorreformatorischer Zeit, nämlich dem frühen I5. Jahrhundert, deutschsprachige Übertragungen belegt. In zahlreichen Kirchen- und Begräbnisordnungen des I6. und I7. Jahrhunderts wird der deutsche Lutherische Gesang für Leichenbegängnisse empfohlen. Textlich thematisiert er eine prekäre Bedrohungssituation des Menschen: Mitten im Leben wird er der Todesnähe gewahr (»mit dem todt vmbfangen«), fürchtet die Hölle und erkennt die Gefahren der Sünde. Durch Anrufung Gottes wird um Beistand

30 Ferner stand er in Kontakt mit dem Superintendenten Peter Streuber aus Sorau. Vgl. Grimm, Meister der Renaissancemusik (wie Anm. I8), S. I95.

3 I Vgl. Kerala J. Snyder, Dieterich Buxtehude. Leben - Werk-Aufführungspraxis, 3. Aufl., Kassel u. a. 2007, S. I7I. Zur Person Andreas Musculus siehe ferner Ernst Koch, »Andreas Musculus und die Konfessionalisierung im Luthertum«, in: Die lutherische Konfessionalisierung in Deutschland, hrsg. von Hans-Christoph Rublack, Gütersloh I992, S. 250-273.

32 Siehe etwa Lucas Lossius und Andreas Musculus, Trostschrifft $\|$ Fuer die jheni=\|gen/Welchen jhre || Vetter / Muetter/Ehegemabel/Kin=\|der/Brueder/Schwester/ vnnd andere gute Freund / auß disem leben verscheiden [...], Frankfurt am Main 1574.

$33 \mathrm{Vgl}$. Anm. 29.

34 Vgl. dazu u. a. Walter Lipphardt, " >Mitten wir im Leben sind ‘. Zur Geschichte des Liedes und seiner Weise «, in: Jabrbuch für Liturgik und Hymnologie 8 (I963), S. 99-II8, sowie: Liederkunde zum Evangelischen Gesangbuch, hrsg. von Gerhard Hahn und Jürgen Henkys, Heft 9, Göttingen 2004, S. 69-78; Michael Fischer, Rebecca Schmidt, "Mein Testament soll seyn am End". Sterbeund Begräbnislieder zwischen I500 und 2000 (Volksliedstudien, 6), Münster 2005, S. 25-66. 


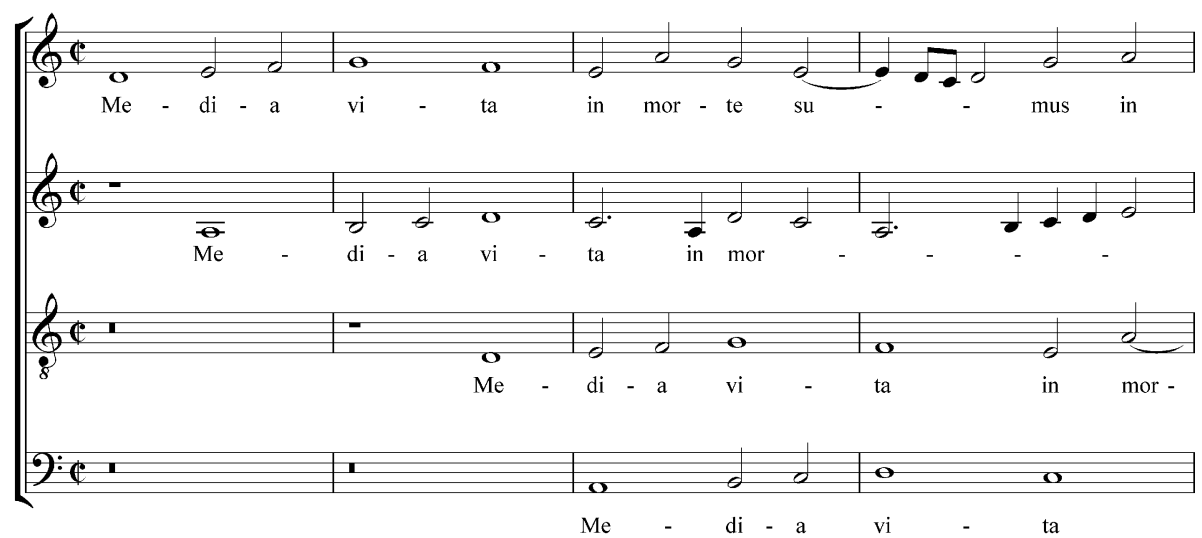

Notenbeispiel I: Gregor Lange, Media vita, Mensur I-4

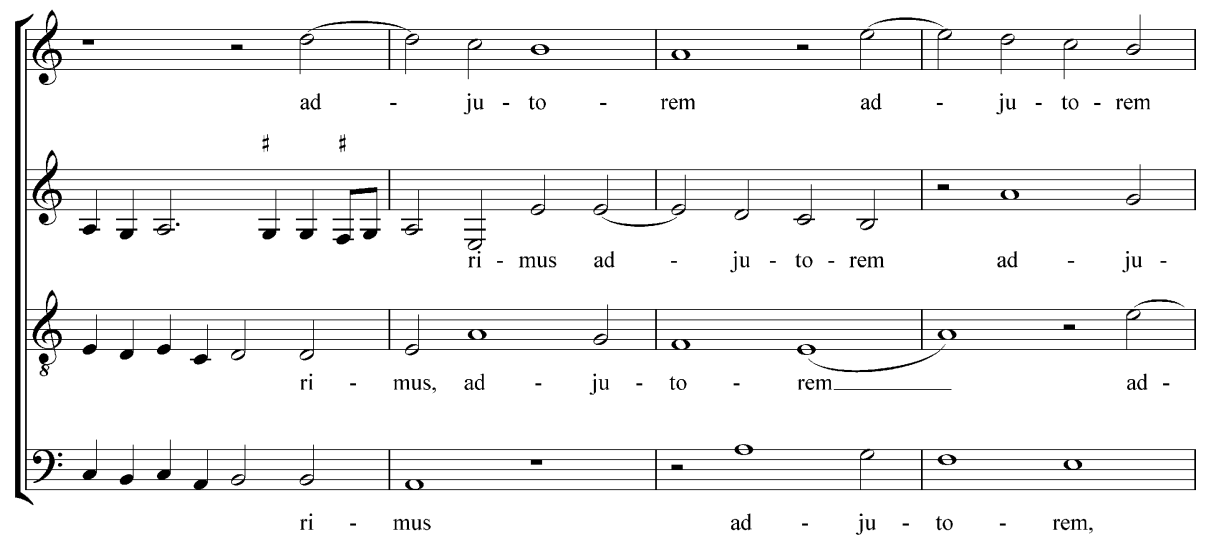

Notenbeispiel 2: Gregor Lange, Media vita, Mensur I6-I9

und Schutz gebeten. ${ }^{35}$ Ludwig Senfl hat in seiner fünfstimmigen Vertonung übrigens den lateinischen und deutschen Text miteinander kombiniert. Welche der zahlreichen Fassungen der Antiphon Gregor Lange in seinem Media-vita-Satz verwendet hat, ist nicht bekannt. Jedenfalls erscheint die Melodie nicht als cantus firmus in nur einer Stimme, sondern wird im vierstimmigen Satz frei verarbeitet. Nach imitatorischem Beginn entwickelt sich ein ausgesprochen ruhevoller Satz.

Das anfänglich aufsteigende Viertonmotiv im Ambitus einer Quart erfährt ab Mensur I6 (»adjutorem«) eine Umkehrung in allen Stimmen und wird klanglich verwoben (siehe Beispiel I und 2). Zentral erscheint die homophone Deklamation

35 Fischer, Schmidt, »Mein Testament soll seyn am End« (wie Anm. 34), S. 27. 


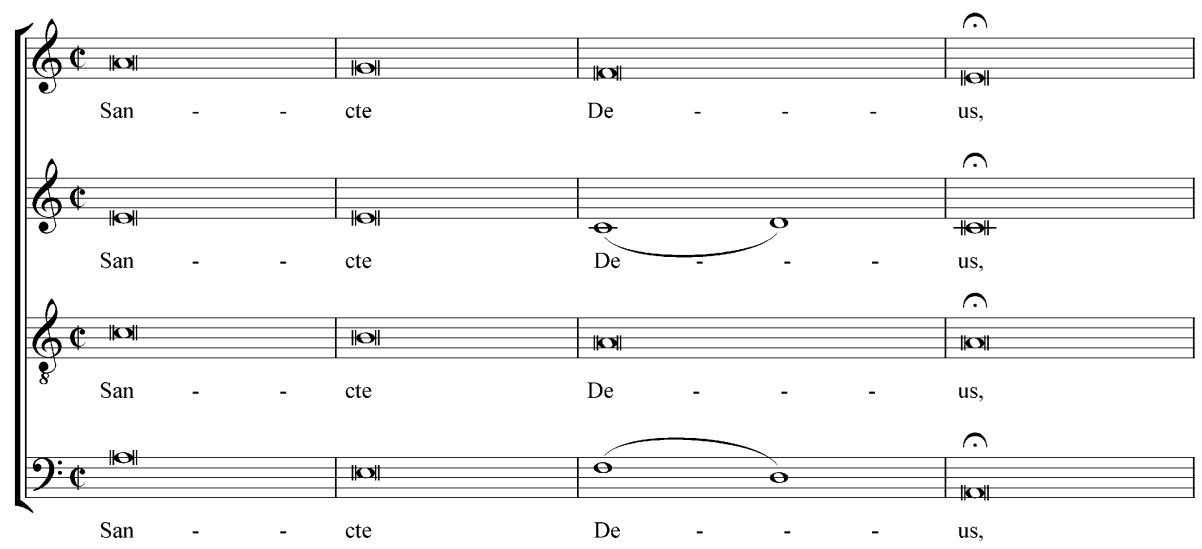

Notenbeispiel 3: Gregor Lange, Media vita, Mensur 34-37

des »Sancte deus, sancte fortis« ab Mensur 34 (siehe Beispiel 3). Hier ist in gedehnter Form erneut das absteigende Quartmotiv im Diskant auszumachen. Die Komposition weist - etwa mit Blick auf die Imitationstechnik - gewisse Ähnlichkeiten zu der freilich sechsstimmigen, bereits 1573 im Druck erschienenen Vertonung von Orlando di Lasso auf. ${ }^{36}$ Die »Sancte deus «-Passage wird von Lasso jedoch gänzlich anders, nämlich als secunda pars ausgesprochen polyphon vertont.

Fraglos weist die hier angesprochene Motette Lange bereits als versierten Komponisten aus, und man mag nachvollziehen können, weshalb er (mutmaßlich) von Breslauer Musikliebhabern zur Übersiedlung bewogen wurde. Gewisse Bande waren bereits insofern nach Breslau geknüpft, als der erwähne Motettendruck von I580 dem Rat der Stadt dediziert ist. Führen wir uns rasch vor Augen, welche Situation Lange in Breslau vorfand: In der wirtschaftlich prosperierenden Stadt lebten im I6. Jahrhundert zwischen zwanzig- und dreißigtausend Menschen; die mehrfach grassierende Pest kostete allein im Jahr 1585 Tausende das Leben. ${ }^{37}$ Zum Vergleich: Langes vormaliger Wirkungsort Frankfurt/Oder zählte lediglich vierbis fünftausend Einwohner. Nach dem Tod König Ludwigs II. fiel Schlesien bekanntlich 1526 aufgrund des Erbfolgevertrages an das Haus Habsburg. ${ }^{38}$ Große Teile der Region waren bereits in den Jahren zuvor protestantisch geworden. In

36 In der Sammlung Patrocinium musice, prima pars, München I573. Bei Lasso erscheint der cantus firmus in langen Noten im Tenor und wird in den übrigen Stimmen imitiert.

37 Vgl. Remigiusz Pośpiech, »Breslau als Zentrum der Musikkultur Schlesiens im I7. Jahrhundert«, in: Schütz-Jabrbuch 32 (2010), S. 7-I6, hier S. 7.

38 Ludwig II. fiel in der Schlacht bei Mohács (1526) gegen die Türken, wodurch die böhmische Königswürde an Ferdinand I. und somit an die Dynastie der Habsburger kam. Vgl. Lothar Hoffmann-Erbrecht, Art. »Breslau«, in: Schlesisches Musiklexikon, hrsg. von Lothar HoffmannErbrecht, Augsburg 200I, S. 6I. Zur Entwicklung der Reformation in Schlesien siehe auch Norbert 
Breslau selbst wirkten mit Johann Heß, seit 1523 Pfarrer an St. Maria Magdalena, und Ambrosius Moibanus, seit I525 Prediger an St. Elisabeth ${ }^{39}$ (also der ehemaligen Wirkungsstätte Thomas Stoltzers), namhafte Reformatoren. Abgesehen vom weiterhin katholischen Domkapitel bekannten sich die Breslauer Kirchen mehrheitlich zur Lehre Martin Luthers. Ein entscheidendes Ereignis war in diesem Zusammenhang die Disputation von Heß und dem Dominikaner Leonhard Cziper im April I524. Mit Blick auf die Bedeutung der Messe vertrat Heß einen eindeutigen Standpunkt:

»Hierauff mag die Meß vnnd das selbig Ampt kayn opffer seyn, denn also hett Christus zu offtmal von anfang der well müssen leyden, sterben, vnnd gemartert werden, Sondern die meß ist allain ain gedechtnus des aynigen Opffers vnnd Testaments durch den Priester vnd opffer volbracht. $« 40$

Das Stadtregiment pflichtete dem Reformator bei und es kam zu diversen Abschaffungen innerhalb der Liturgie, die der Chronist Nikolaus Pol wie folgt zusammenfasste:

»Den 23. Aprilis, am Sonntage Quasimodogeniti ward in den Pfarrkirchen, ohne einigen Tumult, abgeschafft und unterlassen das Anbeten und die Verehrung der Bilder, die Prozession mit dem Sakrament, Vigilien, Seelmessen, Requiem, Anniversarien, Weisung des Heiligthums, Weihung des Wassers, Gewürzes, Salzes, Kräuter. Man fing an Deutsch zu taufen. Allen Priestern ward erlaubet in Ehestand zu treten, und die Kirchenämter und Zeremonien zu verrichten. Es ward zugelassen, am Freitage und in der Fasten Fleisch zu essen. Herr Hans Schnabel, Altarist und Organist zu St. Elisabet, war der erste unter den Priestern, so ein Weib genommen. $\ll^{41}$

So vollzog sich der Übertritt in bemerkenswert ruhiger Weise. Der konfessionelle Frieden blieb wohl auch deshalb erhalten, weil die Reformideen in einer Weise

Hampel, Deutschsprachige protestantische Kirchenmusik Schlesiens bis zum Einbruch der Monodie, Breslau I937, S. IO-I7.

39 Zum Wirken von Ambrosius Moibanus an St. Elisabeth vgl. Jan Harasimowicz, Schwärmergeist und Freibeitsdenken. Beiträge zur Kunst- und Kulturgeschichte Schlesiens in der Frühen Neuzeit (Neue Forschungen zur schlesischen Geschichte, 2I), Köln u. a. 20IO, S. 8I-83.

40 Zitiert nach Quellenbuch zur Geschichte der evangelischen Kirche in Schlesien, hrsg. von Gustav Adolf Benrath u. a., München I992, S. I6.

4I Nikolaus Pol, Jahrbücher der Stadt Breslau, Bd. 3, Breslau I8I9, S. 38. Zu den Klagen der Altaristen von St. Maria-Magdalena beim Domkapitel siehe Hans-Adolf Sander, Beiträge zur Geschichte des Lutherischen Gottesdienstes und der Kirchenmusik in Breslau. Die lateinischen Haupt- und Nebengottesdienste im I6. und 17. Jahrhundert, Breslau I937, S. 4. 
vorgetragen wurden, die eine $»$ offene Konfrontation $«{ }^{42}$ mit der alten Kirche vermied. Mithin kamen auch auf kirchenmusikalischem Sektor kaum Spannungen auf. Man stützte sich weitgehend auf ein Repertoire, das - wie Lothar HoffmannErbrecht gezeigt hat - »vorwiegend von der lateinisch textierten Messe und Motette spätniederländisch-italienischer oder auch deutscher Provenienz bestimmt wurde. «Die »enge Literaturgemeinschaft « zwischen Katholizismus und Protestantismus - so der Schlesienexperte weiter - sei in dieser Zeit ohnehin noch sehr ausgeprägt. ${ }^{43}$ Das erste schlesische protestantische Gesangbuch erschien im Übrigen in der Breslauer Offizin von Adam Dyon im Jahr I525. ${ }^{44}$ Es dokumentiert die Einführung des deutschsprachigen Gemeindesgesangs in den Gottesdienst, ${ }^{45}$ wiewohl die »tatsächliche Beteiligung der jungen lutherischen Gemeinden des mittelund ostdeutschen Sprachraumes am Gesang in den Kirchen ${ }^{46}{ }^{46}$ in der jüngeren Forschung kontrovers diskutiert wird. Hans-Adolf Sander hat dargelegt, dass es in Breslau zu einer Verknüpfung der lateinischen formula missae und der deutschen Messe kam. ${ }^{47}$ Die städtische Kirchenordnung des Jahres 1557 weist darüber hinaus auch auf die polnische Situation hin. So heißt es darin: »So wird zu S. Christoff auch eine polnische Predigt des morgens samt der messe und nach tische wider eine vor das polnische volk, des vil mit uns in der stadt ist, gehalten. ${ }^{48}$ Die ver-

42 Jörg Deventer, Gegenreformation in Schlesien. Die habsburgische Rekatholisierungspolitik in Glogau und Schweidnitz 1526-I707 (Neue Forschungen zur schlesischen Geschichte, 8), Köln u. a. 2003, S. 49 .

43 Vgl. Hoffmann-Erbrecht, Art. »Breslau« (wie Anm. 38), S. 66.

44 Vgl. dazu Das älteste Gesangbuch Schlesiens - Breslau I525, hrsg. und mit einem Kommentar versehen von Anna Manko-Matysiak, Breslau 2004.

$45 \mathrm{Zu}$ den Entsprechungen zwischen jenem Gesangbuch und dem Enchiridion des Erfurter Druckers Mathes Maler vgl. Fritz Feldmann, Die schlesische Kirchenmusik im Wandel der Zeiten, Lübeck I975, S. 24.

46 Hans-Otto Korth, Rezension zu Das älteste Gesangbuch Schlesiens [...] (vgl. Anm. 44), in: Musikgeschichte in Mittel- und Osteuropa, Heft IO, Leipzig 2005, S. 292. Zum »Eindringen« der einstimmigen und mehrstimmigen deutschen Kirchenmusik in den Breslauer Gottesdienst vgl. Hampel, Deutschsprachige protestantische Kirchenmusik (wie Anm. 38), S. 29-30.

47 Sander, Beiträge zur Geschichte des Lutherischen Gottesdienstes (wie Anm. 4I), S. 9ff.; hingewiesen wird in diesem Zusammenhang häufig auf die spezifische Breslauer Form der Matura, »die eine Verschmelzung von Abendmahlshandlung mit der Matutin« darstelle. Andrzej Wolansky/ Fritz Feldmann, Art. »Breslau«, in: Die Musik in Geschichte und Gegenwart, 2. neubearb. Ausgabe, hrsg von Ludwig Finscher, Sachteil Bd. 2, Kassel I995, Sp. I49. In späteren Jahren nahm man offenbar dennoch eine fortschreitende "Auflösung der lateinischen Liturgie«, die insbesondere in den Nebengottesdiensten gepflegt wurde, wahr. Vgl. Hoffmann-Erbrecht, Musikgeschichte Schlesiens (wie Anm. 2), S. 63. Johann Knöfels Cantus choralis ist wiederholt dahingehend interpretiert worden, dieser Entwicklung entgegenwirken zu wollen. Bei der Sammlung handelt es sich um mehrstimmige Ordinarium- und Proprium-Teile, Prosen, Hymnen und Psalmverse durch das Kirchenjahr, die der aus Lauban stammende Komponist dem Rat der Stadt Breslau I575 widmete. Vgl. Feldmann, Die schlesische Kirchenmusik (wie Anm. 45), S. 40-52.

48 Bericht über die kirchliche Ordnung von Breslau I557, zitiert nach: Quellenbuch zur Geschichte der evangelischen Kirche in Schlesien (wie Anm. 40), S. 47. 
gleichsweise moderaten Erneuerungen in Repertoire und Liturgie dürften Lange vertraut gewesen sein. Schenkt man der Märkischen Kirchenordnung vom II. September I540 Glauben, so brachte die nachreformatorische liturgisch-musikalische Praxis auch in seinem früheren Wirkungsort Frankfurt keine wesentlichen Veränderungen. ${ }^{49}$ Mit Blick auf Breslau weist der bereits zitierte Chronist Nikolaus Pol im Übrigen erst für das Jahr von Langes Ankunft - also I583 - auf die Einführung deutscher Lieder an St. Maria Magdalena hin: »Den 24. November [I583], den 26. Sonntag Trinitatis', fing man an, zu St. Maria Magdalena, vor der Mittagspredigt, teutsche Lieder zu singen. ${ }^{50}$

Inwiefern Lange überhaupt aktiv am Musikleben Breslaus partizipierte - es wurde institutionell v. a. von den protestantischen Stadtkirchen St. Elisabeth, St. Maria Magdalena, St. Berhardin bzw. deren Filial- und Nebenkirchen, ${ }^{1}$ dem katholischen Domkapitel ${ }^{52}$ sowie den Klöstern der Augustinerchorherren, Prämonstratenser und Kreuzherren mit dem roten Stern getragen ${ }^{53}$ (auch das vielgelobte Schulwesen ${ }^{54}$ war in die Musikkultur eingebettet) - , inwiefern Lange hier also in Erscheinung trat, ist fraglich. Bekannt ist nur, dass er aufgrund seines Gesundheitszustandes im St. Hieronymus-Hospital lebte. Dabei handelte es sich eigentlich um ein Schülerhospital, das mit dem Eremiten-Kloster St. Dorothea in Verbindung stand, in dem aber auch ältere Menschen Aufnahme fanden. In der Vorrede zum zweiten Teil seiner Cantiones sacrae, die 1584 bei Andreas Wolcken in Nürnberg erschienen, bezeichnet Lange die Musik explizit als »medicina ${ }^{55}$ Die Wahl einzelner Texte in dieser Sammlung und das enge Wort-Ton-Verhältnis bzw. die Fülle musikalisch-rhetorischer Figuren haben denn auch zu Interpretationen geführt, die in den Motetten v. a. eine Selbstreflexion der persönlichen Leidenssituation erblickten. »Es kommen ausserdem darin Wendungen vor, die selbst unsern modernen, Hyperdissonanzen vertragensollenden Ohren für den Gesang bedenklich erscheinen. - heißt es bei Reinhold Starke über die Motette Vae misero mibi (Weh mir Elenden). ${ }^{56}$ Das Stück weist in der Tat einige gewagte Modulationen,

49 Allihn, Art. »Frankfurt an der Oder« (wie Anm. 23), Sp. 667.

50 Zitiert nach Jabrbücher der Stadt Breslau (wie Anm. 4I), Bd. 3, Breslau I82I, S. II5.

5 I St. Barbara, St. Christophorus, St. Trinitatis, Elftausend-Jungfrauen, St. Salvator.

52 V.a. Bischof Andreas Jerin (I585-I596) galt als Förderer der Musik. Zu den sechs Kirchen auf der Dominsel vgl. Barbara Wiermann, Die Entwicklung vokal-instrumentalen Komponierens im protestantischen Deutschland bis zur Mitte des I7. Jahrhunderts (Abhandlungen zur Musikgeschichte I4), Göttingen 2005 , S. 340.

53 Ebd., S. 339 und Pośpiech, »Breslau als Zentrum der Musikkultur« (wie Anm. 37), S. 7.

54 Stadtschulen bei St. Elisabeth und St. Maria Magdalena, Lateinschule an St. Bernhardin.

55 Gregor Lange, Liber secundus cantionum sacrarum, cum adjuncto in fine dialogo, decem vocum, Nürnberg I584.

56 Vorwort zu Langius, Eine ausgewählte Sammlung Motetten (wie Anm. IO), S. IV. 


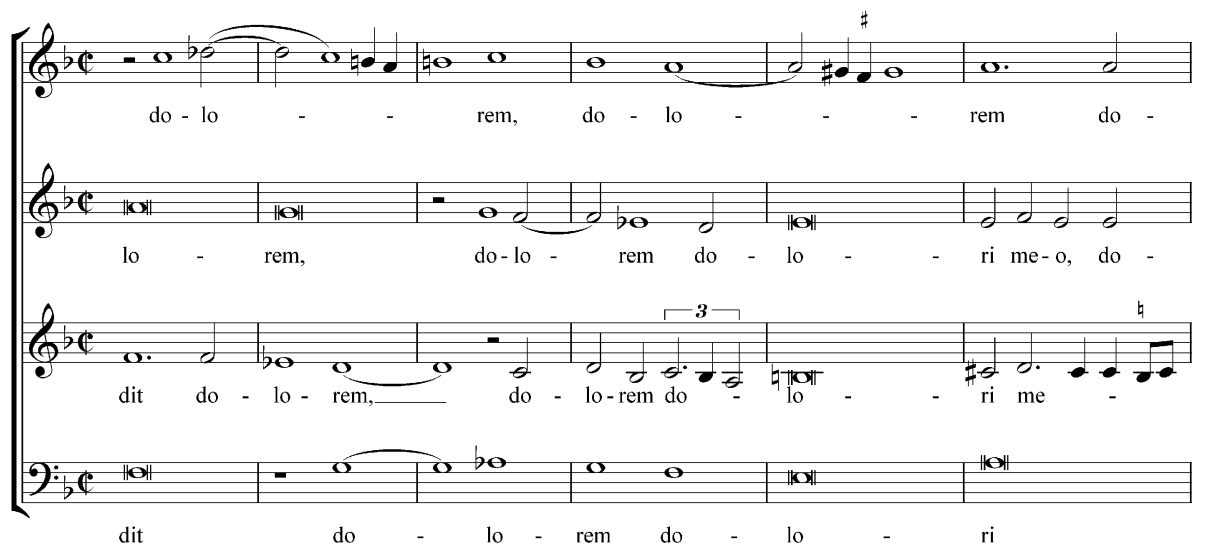

Notenbeispiel 4: Gregor Lange, Cantiones sacrae (I584), Vae misero mihi, T. I4-I9

Durchgangsdissonanzen, tonartfremde Halbtöne (Pathopoeia) und chromatische Passagen auf - wie hier an der Textstelle »dolorem « zu ersehen (Beispiel 4) ${ }^{57}$

Die wehklagenden Worte aus Jeremias Kapitel 45 Vers 3 mögen den angesprochenen Deutungen (zumal im Kontext der vom Autor verfassten Vorrede) Nährboden gegeben haben. Hans Joachim Moser sprach gar vom »spürbar selbsterlebte[n] Vae misero «, ${ }^{58}$ für Lothar Hoffmann-Erbrecht spiegelt das Stück den »Gemütszustand des Gelähmten und Todgeweihten wider «. ${ }^{59}$

Trotz angeschlagener Gesundheit scheint Lange in seinen letzten vier Lebensjahren viel komponiert zu haben. Jedenfalls wurden nun mehrere seiner Werke im Druck veröffentlicht. Wie viele Opera möglicherweise bereits in Frankfurt entstanden sind, kann derzeit nur spekuliert werden. Mit Blick auf die erwähnten Cantiones sacrae II schreibt Lange jedoch in der Vorrede, dass diese größtenteils in Breslau - »in dieser meiner Zwangsanstalt «, also dem Hieronymus-Hospital komponiert seien und er sie "gleichsam als Frucht « den Einwohnern der Stadt zurückgeben wolle. $^{60}$ In der Breslauer Offizin von Johann Scharffenberg fanden innerhalb zweier Jahre folgende Werke ihre Drucklegung:

57 Vgl. dazu auch Feldmann, Die schlesische Kirchenmusik (wie Anm. 45), S. 57-58 und Nils Giebelhausen, Art. »Gregor Lange«, in: Die Musik in Geschichte und Gegenwart, 2. neubearb. Ausgabe, hrsg. von Ludwig Finscher, Personenteil Bd. IO, Kassel 2003, Sp. II65.

58 Hans Joachim Moser, Geschichte der deutschen Musik von den Anfängen bis zum Beginn des Dreißigjührigen Krieges, Hildesheim I968, S. 487.

59 Hoffmann-Erbrecht, Art. »Breslau« (wie Anm. 38), S. 66.

60 Deutsche Übersetzung zitiert nach Starke, »Hieronymus Gregorius Langius Havelbergensis « (wie Anm. 2), S. I09. In der Vorrede weist Lange ferner darauf hin, dass die Cantiones sacrae I weite Verbreitung gefunden hätten. 
- Nuptiis [...] Henrici Schmid. Cantio gratulatoria, Breslau I584.

- Newer Deudscher Lieder mit dreyen Stimmen [...]. Der Erste Theil, Breslau I584.

- Bekenntnis der Sünden, und Gebet umb gnedige Linderung der vorstehenden Not und Gefahr, Breslau I585.

- Prudens simplex: simplex, prudens. Symbolum Francisci Virlingi, Breslau I585.

- Epithalamion melos [...] Iohanni Hennmanno, Breslau I586.

Insbesondere die beiden angeführten Gelegenheitswerke zu Hochzeiten dürften für Lange ein finanzielles Zubrot bedeutet haben; sie lassen zudem auf (vorerst freilich nicht genauer zu bestimmende) gesellschaftliche Kontakte schließen. Der zweite Teil der Neuen Deutschen Lieder ${ }^{61}$ wurde übrigens 1586 in der Breslauer Druckerei von Georg Baumann veröffentlicht. Die Druckhäuser Scharffenberg und Baumann waren familiär verknüpft. Beide Teile der Deutschen Lieder wurden zugleich in dem schon erwähnten Nürnberger Haus von Andreas Wolcken verlegt, der etwa auch mit wichtigen Leipziger Druckern wie Abraham Lamberg zusammenarbeitete. Zwar kommt Breslau im I6. Jahrhundert nicht annähernd die Bedeutung Nürnbergs innerhalb des Druckwesens zu, doch waren auch hier einige wichtige Firmen, die z.T. auch Musikalien produzierten, angesiedelt. Christoph Reske weist in seiner monumentalen Arbeit für unseren Untersuchungszeitraum auf folgende Breslauer Druckereien hin: Konrad Baumgarten, Adam Dyon, Kaspar Libisch, Andreas Winkler d. Ä., Crispin Scharffenberg, Johann Scharffenberg, Georg Baumann.62 Das Beispiel Andreas Wolcken führt zugleich die mitunter engen Geschäftsverbindungen zwischen einzelnen Städten auf dem Felde des Buch- und Notendrucks im I6. Jahrhundert vor Augen. Der bereits im Zusammenhang mit dem Gesangbuch von I525 angesprochene Breslauer Reformationsdrucker Adam Dyon hatte sein Stammhaus übrigens ursprünglich auch in Nürnberg. Weil er seine Druckwerke vorab nicht der Zensur vorlegte, kam es hier jedoch wiederholt zu Streitigkeiten mit dem Rat der Stadt, was Abmahnungen und diverse Strafen nach sich zog und letztlich wohl auch zur Übersiedlung nach Breslau führte (seine dortige Verlagstätigkeit beginnt im Jahr I5I8). ${ }^{63}$ Städtebeziehungen auf Verlagsebene sind auch mit Blick auf Langes vormaligen Wirkungsort Frankfurt/Oder zu verfolgen. Johann Eichhorn d. Ä. - also der Vater von Langes dortigem Drucker Andreas Eichhorn - unterhielt beispielsweise Faktoren in Leipzig, Naumburg,

6I Gregor Lange, Der Ander Theil Newer Deudscher Lieder mit dreyen Stimmen, Breslau 586.

62 Christoph Reske, Die Buchdrucker des 16. und 17. Jahrbunderts im deutschen Sprachgebiet, Wiesbaden 2007, S. I26-I3I. Zu den Druck-Beständen der Breslauer Kirchen zählte u. a. die im Wesentlichen auf italienische Meister ausgerichtete Reihe Sacrae cantiones, die der aus Liegnitz stammende Friedrich Lindner in den 1580 oer-Jahren in Nürnberg herausbrachte. Auch der in fünf Bänden erschienene Thesaurus musicus (bei Montanus und Neuber in Nürnberg verlegt) zählte zu den erworbenen Musikalien. Vgl. dazu Feldmann, Die schlesische Kirchenmusik (wie Anm. 45), S. 37.

63 Reske, Die Buchdrucker (wie Anm. 62), S. I27-I28, 663. Zu Dyons Wirken in Breslau siehe auch Das älteste Gesangbuch Schlesiens, hrsg. von Anna Manko-Matysiak (wie Anm. 44), S. 9-II. 
Danzig, Stettin, Prag und Frankfurt am Main. ${ }^{64}$ Für die Verbreitung von Musikalien sind solche Vertriebsgeflechte durchaus von Relevanz. Langes Drucke wurden im Übrigen auch in diversen Messkatalogen angezeigt. ${ }^{65}$

Neben der Druckveröffentlichung ist freilich auch die handschriftliche Überlieferung zu bedenken. Da die Stadt Breslau Langes letzte Lebensstation war, verwundert es nicht, dass die meisten seiner Kompositionen in den I860er-Jahren Eingang in die Bestände der dortigen Stadtbibliothek fanden ${ }^{66}$, die inzwischen z.T. in Berlin und Krakau verwahrt werden. Zahlreiche seiner Werke sind ferner in Handschriften aus den Sammlungen Löbau, Grimma und Colditz in der Sächsischen Landesbibliothek Dresden greifbar. Den Angaben des Répertoire International des Sources Musicales zufolge befinden sich derzeit in mehreren deutschen, ${ }^{67}$ polnischen, ${ }^{68}$ schwedischen ${ }^{69}$ und englischen ${ }^{70}$ Bibliotheken bzw. Archiven Manuskripte seiner Kompositionen. Mithin ist die handschriftliche Verbreitung von Langes Werken - so viel kann man den (hier nicht weiter spezifizierten) Angaben der heutigen Aufbewahrungsorte entnehmen - durchaus als überregional zu bezeichnen. Freilich lassen sich daraus nur bedingt Aussagen über die ursprüngliche Distribution ableiten. ${ }^{71}$ Erwähnenswert ist v. a. eine Quelle Breslauer Provenienz, nämlich die schon mehrfach erwähnte Handschrift Ms. Mus. I5 aus den ehemaligen Beständen der Breslauer Stadtbibliothek (heute in der Musikabteilung der Staatsbibliothek zu Berlin). Die Handschrift, welche Simon Lyra, seit 1580 Signator und späterer Kantor an St. Elisabeth, zusammengestellt und

64 Vgl. Reske, Die Buchdrucker (wie Anm. 62), S. 269. Die Druckerei von Konrad Baumgarten hatte ihren Ursprung in Breslau.

65 Vgl. Albert Göhler, Verzeichnis der in den Frankfurter und Leipziger Messkatalogen der Jahre 1564 bis I759 angezeigten Musikalien, Hilversum 1965, S. 2I-22 (Göhler I).

66 Die Musikalienbestände stammen ursprünglich v. a. aus den Bibliotheken der Breslauer Kirchen St. Maria Magdalena, St. Bernhardin und St. Elisabeth (Rhedigersche Sammlung). Vgl. dazu Wiermann, Die Entwicklung vokal-instrumentalen Komponierens (wie Anm. 52), S. 344-349, und Rüffler, Die Stadtbibliothek Breslau (wie Anm. 29), S. 89-9I. Zu den Beständen des Akademischen Instituts für Kirchenmusik siehe Ernst Kirsch, Die Bibliothek des Musikalischen Instituts bei der Universität Breslau. Ein Beitrag zur Kenntnis von dem Anteil Schlesiens an den musikalischen Strömungen des I6.-I8. Jahrhunderts, Breslau 1922.

67 Staatsbibliothek zu Berlin - Preußischer Kulturbesitz, Musikabteilung mit Mendelssohn-Archiv; Stadt- und Kreisbibliothek Zittau; Ratsschulbibliothek Zittau; Notenarchiv der St. Katharinenkirche Brandenburg an der Havel; Herzog August Bibliothek Wolfenbüttel; Hochschularchiv der Hochschule für Musik Franz Liszt Weimar; Musikabteilung der Stadtbibliothek Lübeck; Musikabteilung der Bayerischen Staatsbibliothek München; Archiv Evangelisch-lutherisches Pfarramt Udestedt.

68 Biblioteka Jagiellońska Kraków; Biblioteka Gdańska Polskiej Akademii Nauk Gdańsk.

69 Gymnasie- och stiftsbiblioteket i Stadtsbiblioteket Kalmar S-K; Universitetsbibliotek, Carolina Rediviva Uppsala; Musik- och teaterbiblioteket Stockholm S-Skma; Landsarkivet Visby. S-VIl

70 The British Library London; Essex Record Office Chelmsford.

7I Nicht zuletzt ist hier an die Zerstreuung und Zerstörung von Beständen im Zuge der beiden Weltkriege zu denken. 
z.T. selbst niedergeschrieben hat, umfasst 3 IO Stücke und gibt einen Eindruck vom Repertoire, das an dieser Hauptkirche gepflegt wurde. ${ }^{72}$ Mit 49 Werken ist Jacobus Gallus ${ }^{73}$ besonders zahlreich vertreten. Doch bereits an mengenmäßig zweiter Position erscheint mit 35 lateinischen Motetten und deutschen Gesängen Gregor Lange. Damit rangiert er deutlich vor so prominenten Komponisten wie Orlando di Lasso und Giovanni Pierluigi da Palestrina. Dies soll freilich nicht darüber hinwegtäuschen, dass man bei Aufführungen vielfach auf Werke der zuletzt genannten Tonkünstler mit europäischer Ausstrahlung zurückgegriffen hat. In besonderer Weise scheint das Motetten- und Messen-Euvre Lassos die Breslauer Kirchenmusikpflege bestimmt zu haben. Ein Blick etwa auf Ms. Mus. 93, ein Chorbuch aus dem Jahr I568, welches das Repertoire der St. Maria Magdalenen-Kirche widerspiegelt, macht dies deutlich: 8 der insgesamt I6 Kompositionen sind Messen Lassos. ${ }^{74}$ Die Lasso-Rezeption beschränkt sich aber nicht nur auf die zahlreichen in Schlesien überlieferten Handschriften und Drucke, die entsprechende Werkaufführungen nahelegen. Seine Kompositionen dienten mitunter auch als Vorlagen für Parodien. Gregor Langes fünfstimmige Missa super Angelus ad pastores ait Orlandi etwa basiert auf einer 1562 in Nürnberg veröffentlichten (Nr. 8 der Sacrae cantiones) und darüber hinaus in zahlreichen Handschriften überlieferten Motette des langjährigen Leiters der Münchener Hofkapelle. Langes Parodiemesse hat sich - so weit zu sehen ist - in vier Abschriften erhalten:

- Ms. Mus. IO (Nr. 38), Staatsbibliothek zu Berlin - Preußischer Kulturbesitz, Musikabteilung mit Mendelssohn-Archiv

- Ms. Mus. I4 (Nr. 48), Staatsbibliothek zu Berlin - Preußischer Kulturbesitz, Musikabteilung mit Mendelssohn-Archiv

- Mus. ms. 40III, ${ }^{75}$ Biblioteka Jagiellońska Kraków

- Ms. 4005/47, Biblioteka Gdańska Polskiej Akademii Nauk Gdańsk

Es handelt sich bei dem Werk um eine sogenannte lutherische Kurzmesse, bestehend also lediglich aus den Sätzen Kyrie und Gloria. Das gerade erwähnte

72 Vgl. Bohn, Die musikalischen Handschriften (wie Anm. I8), S. 43-52 und Feldmann, Die schlesische Kirchenmusik (wie Anm. 45), S. 34-36.

73 Die reiche Überlieferung von Gallus-Werken dürfte auch mit dessen Verbindung zu Breslau zusammenhängen. Zwischen dem Komponisten und dem Breslauer Bischof Andreas Jerin be stand ein freundschaftliches Verhältnis. I578 hielt er sich auf dessen Einladung hin in Breslau auf. Die Motettensammlung Opus musicum (I586) ist den Bischöfen von Prag, Olmütz und Breslau dediziert. Vgl. dazu Pośpiech, »Breslau als Zentrum der Musikkultur« (wie Anm. 37), S. 9 und Marko Motnik, Jacob Handl-Gallus, Werk - Überlieferung - Rezeption. Mit thematischem Katalog, Tutzing 2012, S. 69-75.

74 Vgl. Feldmann, Die schlesische Kirchenmusik (wie Anm. 45), S. 33.

75 Laut Wolfgang Boetticher ist die Missa hier im »Cantionale III des Caspar Peltsch« überliefert; vgl., Orlando di Lasso und seine Zeit 1532-I594. Repertoire-Untersuchungen zur Musik der Spätrenaissance, Kassel I958, S. 544. Zu Lassos eigenen Parodiemessen vgl. Rufina Orlich, Die Parodiemessen von Orlando di Lasso, München 1985. 


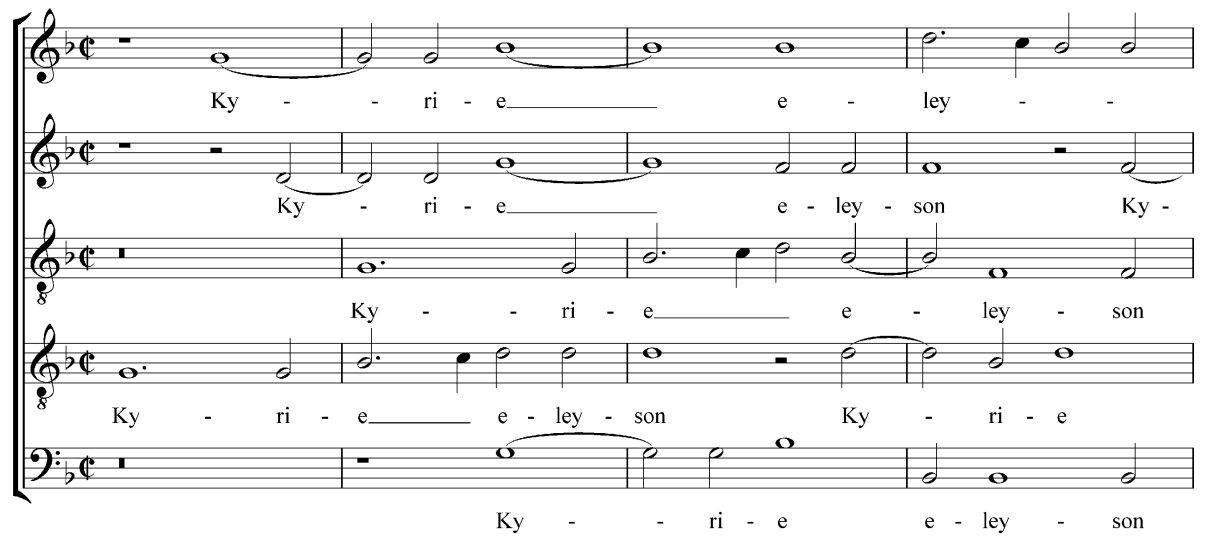

Notenbeispiel 5: Gregor Lange, Missa super Angelus ad pastores ait Orlandi, T. I-4

Chorbuch der St. Maria-Magdalenenkirche (Ms. Mus. 93) zeigt übrigens, dass in Breslau auch von Lasso-Messen nur die ersten 2 bzw. 3 Ordinariumssätze abgeschrieben wurden. Man beschränkte sich hier also gewissermaßen auf die Sätze, die für die Gottesdienstpraxis relevant waren. ${ }^{76}$ Aus Ms. Mus. I4 geht hervor, dass die Lange-Messe passend zum Inhalt der Originalmusik - also der Verkündigung der Weihnachtsbotschaft an die Hirten (Lukas 2, IO-II) - noch Teil der musikalischen Gestaltung des Weihnachtsoffiziums im Jahr I626 war. ${ }^{77}$

Um einen gewissen Eindruck von der Komposition (im Übrigen eine von zwei erhaltenen Messen des Wahlbreslauers ${ }^{78}$ ) zu gewinnen, sei abschließend noch ein kurzer Blick auf das Kyrie geworfen. ${ }^{79}$ Im Sinne der Mottotechnik ist der Anfang der Messe nahezu wörtlich in allen Stimmen von der Vorlage ${ }^{80}$ übernommen worden (Beispiel 5). Im Grunde entfernt sich Lange erst ab Mensur 8 in nennenswerter Weise von der Originalmotette (Beispiel 6).

76 Vgl. Sander, Beiträge zur Geschichte des Lutherischen Gottesdienstes (wie Anm. 4I), S. 89.

77 »Greg. Lange, Intr. Puer natus est nobis et filius $4 \mathrm{v}$.

Missa super: Angelus ad pastores $5 \mathrm{v}$.

Resp. Et cum spiritu tuo. - Amen 4 v.

Joh. Knefel, Alleluja: Dies sanctificatus illuxit $5 \mathrm{v}$.

Prosa. Grates nunc omnes reddamus

Huic oportet ut canamus $5 \mathrm{v}$.

Resp.: Deo dicamus gratias Alleluja 4 v.«

Zitiert nach Sander, Beiträge zur Geschichte des Lutherischen Gottesdienstes (wie Anm. 4I), S. 89.

78 Das zweite Werk trägt den Titel Missa super In dieser weiten Welt.

79 Die nachfolgenden Notenbeispiele basieren auf der Quelle Ms. 4005/47, Biblioteka Gdańska Polskiej Akademii Nauk Gdańsk.

80 Zum Vergleich wurde folgende Edition herangezogen: Orlando di Lasso, The Complete Motets 2, hrsg. von James Erb, Middleton 2002, S. 50-54. 


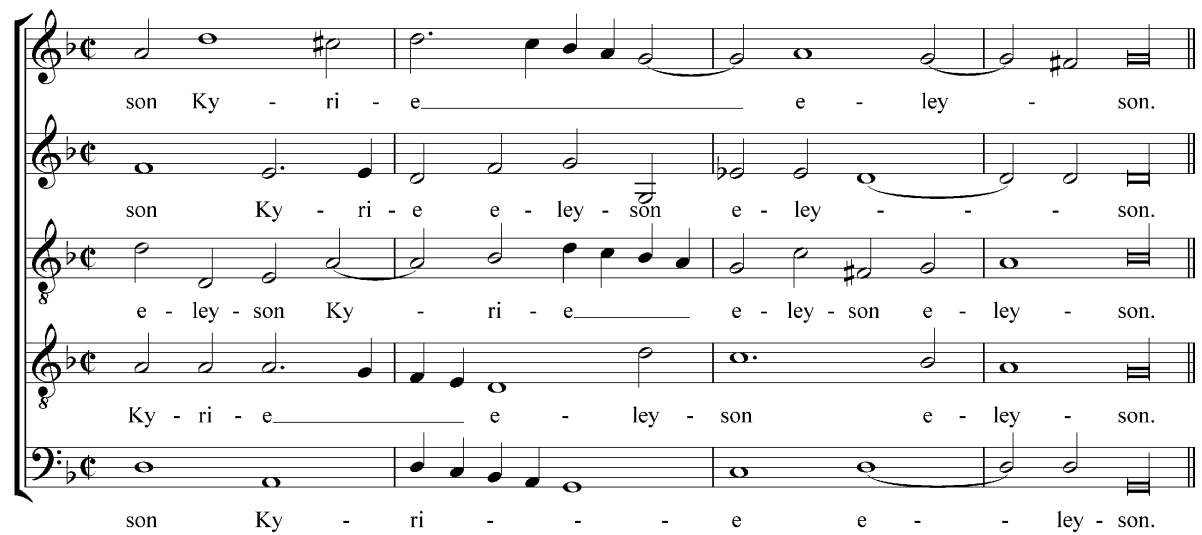

Notenbeispiel 6: Gregor Lange, Missa super Angelus ad pastores ait Orlandi, T. 8-II

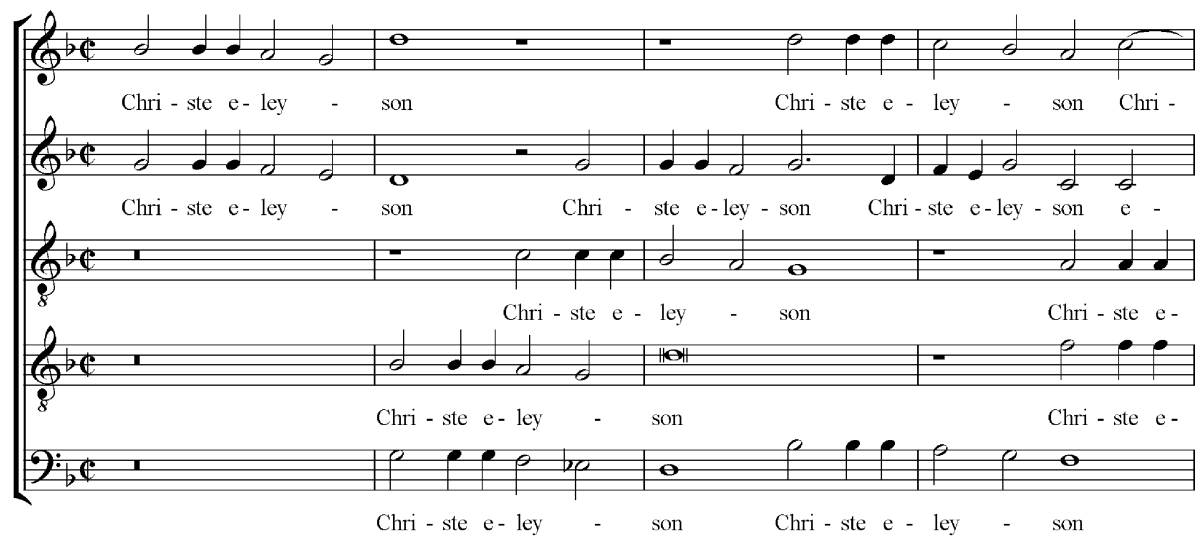

Notenbeispiel 7: Gregor Lange, Missa super Angelus ad pastores ait Orlandi, T. I2-I5

Hier endet bei Lasso der erste Abschnitt; in der Messe setzt die Schlusskadenz des Kyrie I an. Der blockhaft in je zweistimmigen Gruppen anhebende Christe-Teil (Mensur I2) greift zunächst wörtlich den zweiten Motettenabschnitt »Annucio vobis « auf (Beispiel 7). In der Folge häufen sich dann rhythmische und melodische Abweichungen von der Vorlage. Der homophone Schluss des Christe (Mensur 20-24) ist gänzlich frei gearbeitet. Das Kyrie II (Mensur 25) basiert sodann auf dem Motettenabschnitt »qui natus est (Beispiel 8).

Erst mit Beginn der Verarbeitung der »Alleluja«-Takte (durch ein charakteristisches repetierendes Viertonmotiv bestimmt) ist eine >kreativere $<$ musikalische Formbildung auszumachen. Das originäre Material wird nun gleichsam in Versatz- 


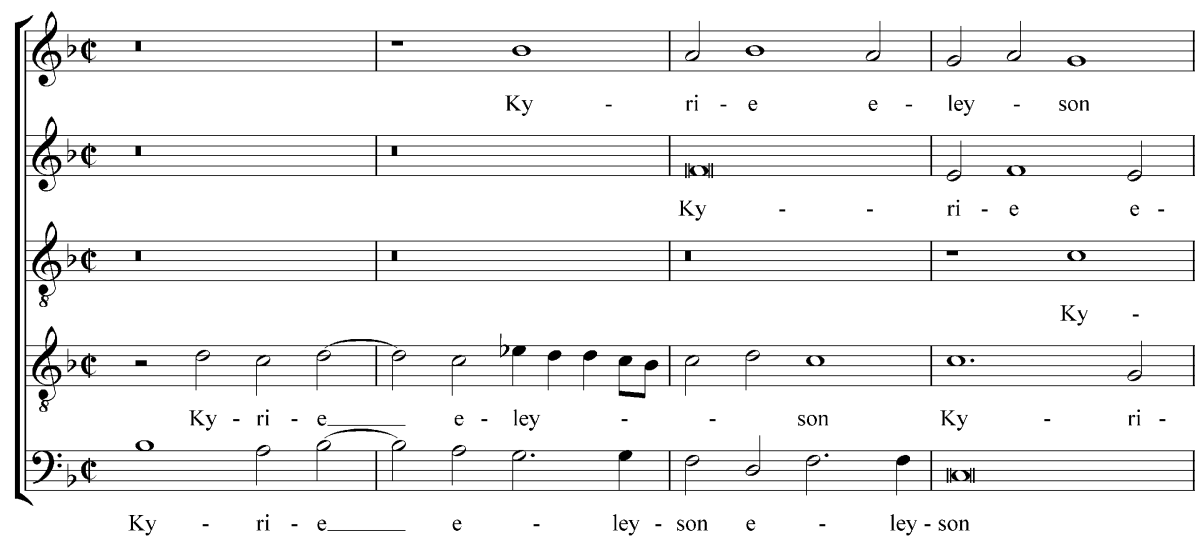

Notenbeispiel 8: Gregor Lange, Missa super Angelus ad pastores ait Orlandi, T. 25-28

stücken mit hinzugefügten und imitierten Teilen kombiniert. Allerdings kommt es nur an zwei Stellen zu einem kurzfristigen Stimmentausch. Ansonsten ist die Bindung der Einzelstimmen an die jeweilige Stimmenvorlage evident. Die vergleichsweise wenigen entlehnungsfreien Abschnitte des Werkes - dies gilt im Wesentlichen auch für das Gloria - machen deutlich, dass der kompositorische Eigenanteil Langes recht gering ist. Parodiemessen über Lasso-Vorlagen waren im späten I6. und frühen I7. Jahrhundert freilich keine Seltenheit. ${ }^{81}$ Hinzuweisen wäre u.a. auf entsprechende Werke von Bartholomäus Gesius, ${ }^{82}$ Basilius Lechmann, ${ }^{83}$ Kaspar Speiser, ${ }^{84}$ Bartholomeus Kellner, ${ }^{85}$ Alexander Utendal ${ }^{86}$ und Jacob Regnart. ${ }^{87}$ Aufmerken lassen v. a. die fünf Vertonungen des (mutmaßlichen) Lange-

8I Zu weiteren Parodien (v. a. Magnificat-Kompositionen) über Lasso-Sätze siehe Boetticher, Orlando di Lasso und seine Zeit (wie Anm. 75), S. 522, 7I4-720, 8I6-8I7.

82 Missa Estote ergo misericordes, Biblioteka Gdańska Polskiej Akademii Nauk, Gdańsk PL-GD (PLGD Ms 4006); Missa Benedicam Dominum (PL-GD Ms 4007); Missa Ecce Maria genuit (PL-GD Ms 4OI3); Missa Deus misereatur nostri (PL-GD Ms 4006); Missa Deus in adjutorium meum intende (PL-GD Ms 4007).

83 Missa super Domine quid multiplicati sunt, Herzog August Bibliothek, Wolfenbüttel D-W (D-W Cod. Guelf. 322 Mus. Hdschr. [Nr. 67] und D-W I5. 2 Musica coll. inc. [Nr. 24]).

84 Missa super Alleluia, Herzog August Bibliothek, Wolfenbüttel D-W (D-W Cod. Guelf. 322 Mus. Hdschr. [Nr. 4] und D-W Cod. Guelf. 32I Mus. Hdschr. [Nr. I4]).

85 Missa super Lauda Jerusalem, Stadsbibliotek, Västerås S-V (S-V Molér 68 [24]).

86 Missa super Surge propera amica, Ratsschulbibliothek, Zwickau D-Z (D-Z Mu 228).

$87 \mathrm{Zu}$ den Parodiemessen über Lasso-Vorlagen von Jacob Regnart vgl. Andreas Waczkat, »Ein ehrenhaftes Spielen mit Musik". Deutsche Parodiemessen des 17. Jahrhunderts, Kassel u. a. 2000 S. 3I6-3I7. Zu weiteren Parodiemessen über Lasso-Motetten von Ivo de Vento und Andrea Gabrieli vgl. Marie Louise Göllner, »Lassos Motetten nach Hymnentexten und ihre Parodiemessen von Ivo de Veno und Andrea Gabrieli «, in: Orlando di Lasso in der Musikgeschichte, hrsg. von Bernhold Schmid, München I996, S. 87-IOO. 
Schülers Gesius, ${ }^{88}$ da er als langjähriger Kantor in Frankfurt/Oder (I593-I6I3) ebenfalls über Verbindungen nach Schlesien verfügte. Hier zeichnen sich möglicherweise gewisse Muster der brandenburgisch-schlesischen Lasso-Rezeption ab. ${ }^{89}$ Das lexikographische Schrifttum hat in Lasso jedenfalls die zentrale Bezugsgröße für Gregor Lange erblickt. In Caspar Theophil Schindlers im Jahr I706 in Liegnitz erschienenen Silesia Togata heißt es denn auch: »Langius Orlandi modulandi proximus arte, Orlandi haut dispar laudis aroma meret. «90

88 Zum möglichen Lehrer-Schülerverhältnis vgl. Waczkat, "Ein ehrenhaftes Spielen mit Musik" (wie Anm. 87), S. 80 .

89 Auch Lange selbst fungierte als Vorlagekomponist für eine Parodiemesse. Der eben erwähnte Bartholomäus Gesius (alias Bartel Göß) schrieb eine fünfstimmige Missa ad imitationem Cantionis Gregorii Langii: Ecce venit rex tuus. Die Motette stammt aus Langes Cantiones aliquot novae (1580). Vgl. Bartholomäus Gesius, Missae ad imitationem cantionum Orlandi et aliorum probatissimorum Orlandi (Elisabeth-Musiquen, 5), hrsg. von Lars Klingberg, Beeskow und Berlin 2009.

90 Caspar Theophil Schindler, Silesia Togata, Liegnitz I706, S. I65. 